Article

\title{
Effects of Virtual Reality Locomotion Techniques on Distance Estimations
}

\author{
Julian Keil *(D), Dennis Edler (D), Denise O’Meara, Annika Korte (D) and Frank Dickmann (D) \\ Geography Department, Cartography, Ruhr University Bochum, 44801 Bochum, Germany; \\ Dennis.Edler@rub.de (D.E.); Denise.Omeara@rub.de (D.O.); Annika.Korte@rub.de (A.K.); \\ Frank.Dickmann@rub.de (F.D.) \\ * Correspondence: Julian.Keil@rub.de
}

check for updates

Citation: Keil, J.; Edler, D.;

O'Meara, D.; Korte, A.; Dickmann, F. Effects of Virtual Reality Locomotion Techniques on Distance Estimations. ISPRS Int. J. Geo-Inf. 2021, 10, 150. https://doi.org/10.3390/ijgi10030150

Academic Editors: Wolfgang Kainz and Norbert Bartelme

Received: 22 January 2021

Accepted: 5 March 2021

Published: 8 March 2021

Publisher's Note: MDPI stays neutral with regard to jurisdictional claims in published maps and institutional affiliations.

Copyright: (c) 2021 by the authors. Licensee MDPI, Basel, Switzerland. This article is an open access article distributed under the terms and conditions of the Creative Commons Attribution (CC BY) license (https:// creativecommons.org/licenses/by/ $4.0 /)$

\begin{abstract}
Mental representations of geographic space are based on knowledge of spatial elements and the spatial relation between these elements. Acquiring such mental representations of space requires assessing distances between pairs of spatial elements. In virtual reality (VR) applications, locomotion techniques based on real-world movement are constrained by the size of the available room and the used room scale tracking system. Therefore, many VR applications use additional locomotion techniques such as artificial locomotion (continuous forward movement) or teleporting ("jumping" from one location to another). These locomotion techniques move the user through virtual space based on controller input. However, it has not yet been investigated how different established controller-based locomotion techniques affect distance estimations in VR. In an experiment, we compared distance estimations between artificial locomotion and teleportation before and after a training phase. The results showed that distance estimations in both locomotion conditions improved after the training. Additionally, distance estimations were found to be more accurate when teleportation locomotion was used.
\end{abstract}

Keywords: virtual reality; artificial locomotion; smooth locomotion; teleportation; distance estimation; spatial cognition

\section{Introduction}

The cognition of geographic space is a topic of rising importance in disciplines dealing with spatial visualization, such as spatial cognition and cartography [1-3]. To shape out an accurate mental representation of the environment, people often use external references [4-6]. These external sources can be cartographic media, such as "traditional" 2D printed maps or interactive and animated digital spatial representations. The improvement of such cartographic media, with the aim of reducing spatial distortions in cognitive representations of space and to increase the efficiency in map-reading performance, has been under study for years [7-9].

Recent advances in hardware and software technology have provided new possibilities for communicating spatial information. Virtual reality (VR) systems allow visualization of (and interaction with) dynamic virtual 3D environments. Immersive features like a first-person view, stereoscopic displays, and the transfer of real-world movement into virtual space (room scale VR) can provide users of VR with a sense of presence unmatched by screen- or paper-based presentation of spatial information [10,11]. Additionally, using already available geospatial data and game engines like Unity or Unreal Engine, VR-based 3D spatial representations of real-world space can be created fast and cost-efficiently [12-15]. Recently, the use of such VR-based spatial representations has become an increasing research topic in a broad range of academic fields operating with spatial data, such as 3D cartography [16,17], laser scanning and photogrammetry [18,19], urban geography and planning [14,20,21], biodiversity [12], glaciology [22], landscape research [23], noise pollution [24], cultural heritage and architecture [25,26], and education $[27,28]$. 
Hruby et al. [29] argue that VR-based spatial representations are "realistic 1:1 replicas you can evaluate concerning individual characteristics as you would do being really there". This makes visualizing space in VR interesting for cases where interaction with virtual space is safer or more cost efficient than experiencing real-world space, e.g., hazard training simulations or "visiting" remote spaces [30]. However, it is important to consider that interaction with a VR environment still differs from real-world interaction. Despite the lack of self-motion cues, VR locomotion techniques such as teleportation or artificial locomotion are applied to a great variety of VR applications. The choice of locomotion techniques may have severe impacts on the spatial cognition within a virtual reality environment [31]. By interacting with a VR environment, people gradually build a mental representation of the displayed geographic space, and the applied locomotion techniques within the virtual model can influence these cognitive representations [32]. This may be due to spatial relations and distances being experienced differently based on the applied locomotion technique.

First studies on the effects of VR locomotion on spatial cognition already indicate that different locomotion techniques lead to different levels of spatial awareness in a virtual reality environment. Coomer et al. [33] suggest arm-cycling as a locomotion technique that causes a high spatial awareness. It is also argued that teleporting could lead to spatial disorientation, because self-motion cues are lacking [34,35]. In terms of directional errors in navigational tasks, continuous locomotion techniques seem to show slight advantages over discrete approaches as teleportation [36]. However, how the lack of self-motion cues of different controller-based VR locomotion techniques affects distance estimations is still not fully understood. Being able to estimate distances accurately is important for the formation of precise cognitive representations of space, because distance estimations are required to assess the spatial relations between elements within a real or virtual environment [37]. Consequentially, if the used VR locomotion technique affects distance estimations, cognitive models created based on interaction with a virtual environment are susceptible to systematic distortions. Campos et al. [38,39] assessed effects of body-based and visual self-motion cues on distance estimations and found that withholding specific self-motion cues deteriorated distance estimations. In this paper, we aim to extend these studies by investigating effects of two of the most commonly used VR locomotion techniques with limited self-motion cues (teleportation and artificial locomotion) on distance estimations in a virtual environment. The results aim to broaden our understanding of spatial perception in VR environments and may help to choose the most appropriate type of locomotion for a VR application based on its defined purpose.

\section{Background}

Distance estimations during locomotion within real-world and virtual space can be distinguished based on the information sources they are linked with. The perception of selfmotion and the assessment of traveled distances can be based on the use and integration of different sensory information as optic flow, proprioception, and vestibular feedback [39-41]. Optic flow is defined as visual information about the relative motion between an observer and the environment [42]. Proprioception describes the perception of self-motion based on information provided by receptors called proprioceptors located in the muscles, joints, and skin $[43,44]$. The vestibular system located in the inner ear provides information about angular and linear acceleration of the head $[45,46]$. Thus, if distances within a VR environment are supposed to be perceived as similar to distances within the real world, VR locomotion techniques should provide similar visual, proprioceptive, and vestibular information as real-world locomotion.

The most natural form of locomotion available in VR environments is room scale locomotion [47]. Room scale VR uses either tracking stations or cameras built into the headmounted display (HMD) to track changes of the position and rotation of the HMD and controllers. Real world movements of the HMD and controllers are then mirrored onto the simulated head position and rotation, as well as the position and rotation of $3 \mathrm{D}$ controller models in virtual space [48]. Thus, similar to real-world locomotion, visual information 
changes based on natural body movements. If the used tracking system is sufficiently accurate, room scale locomotion is hardly distinguishable from natural locomotion in the real world, because the user receives accurate visual, proprioceptive, and vestibular feedback [49].

Unfortunately, the size of the play area available in room scale VR is limited by multiple factors. These include the range of the tracking stations or cameras used to track user movements, the length of the headset wiring (as most VR HMDs are still tethered), and most importantly the size of the used room. Consequentially, if the size of a virtual environment exceeds the size of the available play area, room scale locomotion reaches its limits [50]. In these cases, additional VR locomotion techniques are required. Two of the most popular types of VR locomotion besides room scale are artificial locomotion (also called smooth locomotion or trackpad locomotion) [51] and teleportation. Artificial locomotion is used to apply a steady movement to the location of the user within VR space based on controller input. Usually, an analog stick or a touch pad is used to select the direction or the speed of the movement. Teleportation locomotion is also based on controller input. However, instead of applying a steady movement, a target location is selected by pressing a button and aiming at the location with the controller. When the pressed button is released, the user is instantly transferred to the selected target location.

Both of these locomotion techniques differ from natural movement in the real world, because they lack proprioceptive and vestibular feedback [34,35]. As users of artificial locomotion or teleportation locomotion do not actually travel through real-world space, neither proprioceptors nor the vestibular system are triggered. This lack of specific selfmotion cues can affect how VR space is perceived. For example, Campos et al. [38,39] found that distance estimations tend to be underestimated when distances are only visually perceived without proprioceptive or vestibular locomotion feedback. Additionally, distance estimations of traveled distances only based on visual information were found to be shorter compared to distance estimations based on locomotion with proprioceptive and vestibular feedback [39]. Based on these findings, we assume that distances are underestimated when artificial locomotion or teleportation locomotion are used, because they also lack proprioceptive and vestibular feedback. Concerning teleportation, an additional threat for the accurate perception of locomotion is the lack of optic flow information [52], because, as opposed to continuous forward locomotion, teleportations from one location to another are carried out instantly. As shown in studies of McLeod and Ross [53] and Redlick et al. [54], people can use optic flow information for distance and time to target estimations. Due to the lack of optic flow information, teleportation-based distance estimations require the user to estimate self-to-target distances separately for each teleportation. Multiple distance estimations of consecutive teleportations must then be added up. Therefore, we assume that teleportation-based distance estimations are more error-prone than artificial locomotion-based distance estimations.

Concerning distance estimations in general, they need to be distinguished between distances within peripersonal space, distances within extrapersonal space, or distances between locations in peripersonal space and locations in extrapersonal space. Peripersonal space is the close area within reach of our extremities. Extrapersonal space is the area around our peripersonal space $[55,56]$. First studies on distance perception in VR demonstrate that estimation errors within peripersonal space are small [56,57]. Distances ranging into the extrapersonal space were found to be estimated less accurately with greater variations in the estimations and were more likely to be underestimated [56-58]. In the context of the formation of mental representations of space based on VR locomotion, the focus is on distances ranging into the extrapersonal space, because visual cues as teleport target locations or visual elements providing optic flow information are often outside the peripersonal space. Therefore, the mentioned studies support the already stated assumption that distances are underestimated when artificial locomotion or teleport locomotion are used.

When the accuracy of distance estimations is assessed, it must also be taken into account under what circumstances distances are estimated. Previous studies on distance 
estimations in virtual environments usually used either blindfolded walking towards a target, which has been formerly presented visually in VR, or placing a marker remotely to estimate the distance of a previously traveled path or presented object [38,39,59-62]. In a locomotion context, two additional types of distance estimations are applicable: replicating a previously traveled distance or estimating the metric value of a formerly traveled distance. Replicating traveled distance is important for finding one's way back to a start location. Metric distance estimations can be used to communicate distances or to integrate travelled routes into a complex mental representation of space. Previous studies have shown that people tend to underestimate distances if they have to apply a metric value to them $[56,63,64]$. Although distance estimations in these studies were not locomotion-based, the results support the previously made assumption that teleportation-based distance estimations will be underestimated, because distances are assumed to be assessed based on estimating visual self-to-target distances for each teleportation.

If, as assumed, teleportation and artificial locomotion-based distance estimations are prone to systematic errors, the question arises whether there are ways to reduce these errors. Richardson and Waller [65] demonstrated that systematic underestimations of distances traveled blindfolded (based on previously visually perceived distances in a virtual environment) can be reduced by a training. Concerning metric self-to-target distance estimations in a real-world environment, Allen and Rashotte [66] found that verbal feedback or visual milestones (markers at $50 \mathrm{~m}$ intervals) significantly reduced estimation errors. Based on these findings, we assume that the accuracy of VR-based replicated traveled distances and metric distance estimations can also be improved by a training.

If, as argued, perception of distances in virtual environments is affected by the used locomotion techniques, applying a specific locomotion technique (as artificial locomotion or teleportation) can affect the formation of mental representations of the virtual space. Reducing such potential errors in distance estimations, for example with a training procedure, may help to create more accurate mental representations of virtual environments. In order to experimentally investigate the assumed effects of VR locomotion techniques and training on distance estimations in virtual environments, we formulate the following hypotheses:

Hypothesis 1 (H1). Distances traveled with artificial locomotion or teleportation locomotion in $V R$ are underestimated.

Hypothesis 2 (H2). Distance estimations are more inaccurate when teleportation locomotion is used.

Hypothesis 3 (H3). Distance estimations based on VR locomotion can be improved by training.

\section{Methods}

The study was conducted in accordance with the Declaration of Helsinki. The used study design has been reviewed and approved by the ethics committee of the Faculty of Geosciences at the Ruhr University Bochum.

\subsection{Participants}

The study sample consisted of 40 undergraduate students from Ruhr University Bochum (26 females, 14 males). The age ranged between 18 and 30 years $(\mathrm{M}=23.65$; $\mathrm{SD}=2.47)$. To ensure similar perception of the visual stimuli displayed in the experiment, normal or corrected vision was defined as a precondition for participation.

\subsection{Materials}

Using the game engine Unity (Unity Technologies, San Francisco, CA, USA, version 2019.1.0f2), a virtual 3D environment was built. This environment contained a long corridor without a ceiling (see Figure 1). The floor was covered with two different textures. A path in the center of the corridor with a width of 1 virtual meter was covered with a sand texture. 
The rest of the floor was covered with a shrub texture. These visual patterns were meant to enable the perception of movement by providing optic flow information. To prevent counting strategies based on visual stimulus input, focus was placed on avoiding repetitive patterns in the used textures. As pretests showed that complex textures in the peripheral field of view increased VR sickness, the walls confining the corridor were covered with a solid (thus minimalistic) green texture.

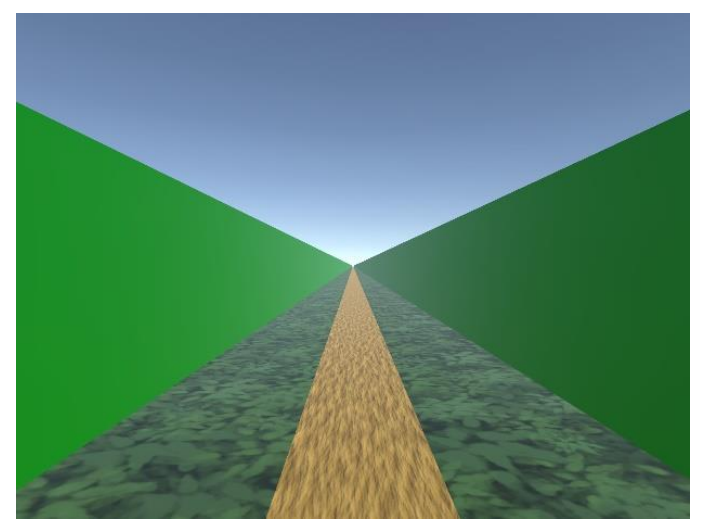

Figure 1. 3D model of a corridor used for distance estimations. The ground was covered with a sand and a shrub texture to enable the perception of movement. For the walls, a solid green color was used to minimize the risk of VR sickness.

To implement VR support, the SteamVR asset developed by Valve was used. This asset enables the use of a great variety of VR HMDs (e.g., HTC Vive, HTC Vive Pro, Valve Index) and controllers in Unity projects. It also provides room scale tracking in Unity Projects when a compatible HMD is used. Additionally, SteamVR adds 3D models of the used controllers and hand models to the virtual environment. The fingers of the hand models move based on controller input.

Two locomotion techniques, teleportation and artificial locomotion, were implemented. Teleportation enabled users to instantly change their current location in the virtual environment using controller input (see Figure 2, left side). Pressing down on a touchpad activated an arc. The location where the arc touched the ground indicated the teleportation target. By adjusting the position and rotation of the controller, users were able to change the angle of the arc and consequentially the teleportation target. When the pressure on the touchpad was released, users were instantly teleported to the target location.
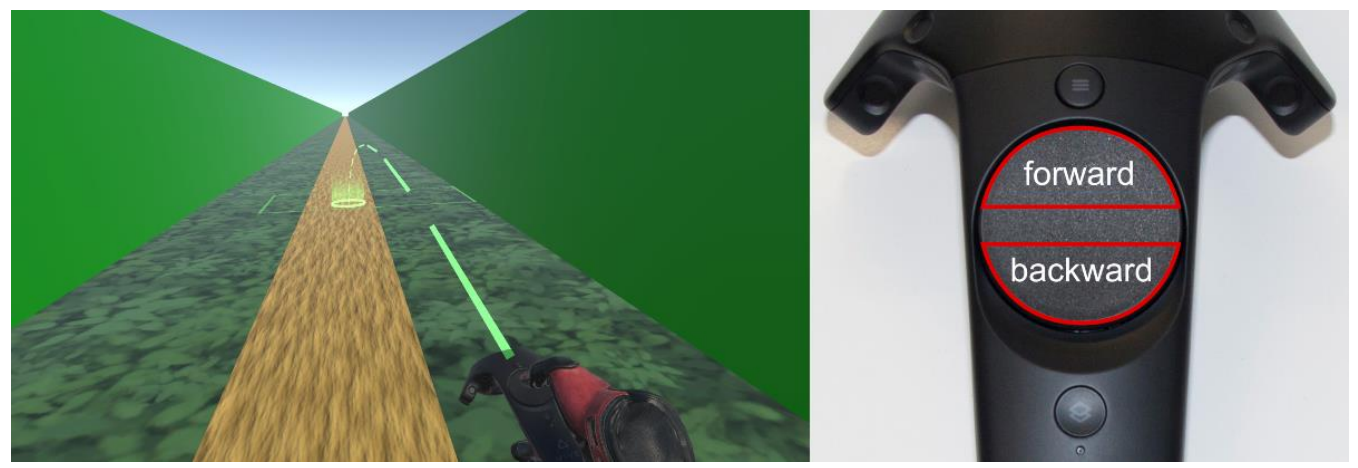

Figure 2. Implemented VR locomotion methods compared. The left side shows the teleportation function. Aiming with an arc that originated from the used controller allowed users to select a teleportation target. The right side demonstrates the controller assignment for the artificial locomotion. The upper $40 \%$ of the touchpad was assigned to a continuous forward movement, the lower $40 \%$ to a continuous backward movement. 
Artificial locomotion was controlled with touchpad input. The top of the touchpad (upper $40 \%$ ) was linked to a continuous forward movement in the virtual environment with a fixed speed of $5 \mathrm{~km} / \mathrm{h}$. Touching the bottom of the touchpad (lower $40 \%$ ) continuously moved the user backward in the virtual environment with the same speed (see Figure 2, right side). The center of the touchpad ( $20 \%$ of the vertical size) was not linked to a movement function. This was meant to prevent an undesired switch of the movement direction.

\subsection{Procedure}

All experiments of the study were carried out in a quiet laboratory equipped with SteamVR Lighthouse tracking stations. Before the experiment started, participants were informed about the procedure and were asked to give signed informed consent. Each participant was then assigned to one of the two investigated VR locomotion techniques (teleportation or artificial locomotion). For each participant, the experimental procedure was carried out twice (once with each VR locomotion technique), but the order of used VR locomotion technique was randomized (crossover design). Participants were asked to stand in the center of the room and were equipped with an HTC Vive Pro HMD and an HTC Vive Controller (see Figure 3). In order to allow accommodation to the VR space, the first visualized scene was a large empty plane with a checkerboard pattern on the floor. VR locomotion was disabled in this scene. After the participants felt accommodated and the HMD was fitted correctly and comfortably, the experimenter manually switched to the next scene. This scene showed the corridor from Figure 1 with a red dot on the floor (see Figure 4 ) at a distance of 6.87 virtual meters from the start position. To train the use of the assigned locomotion technique, participants had to navigate towards the red dot. The distance was chosen randomly, but was similar for all participants, to prevent a training of distance estimations. After participants reached the red dot, a similar scene was displayed, but the red dot was located at a random distance between 90 and 120 virtual meters from the start position. Participants were asked to use the assigned locomotion technique to reach the red dot and to estimate its distance from the start position (distance visible target). The minimum distance of the red dot was selected to ensure that the dot was not visible from the start position. This required the participants to estimate the traveled distance instead of applying a visual self-to-target estimation before they used the assigned locomotion technique. The maximum distance was selected to limit the scene duration and consequentially minimize the risk of VR sickness in the artificial locomotion condition.

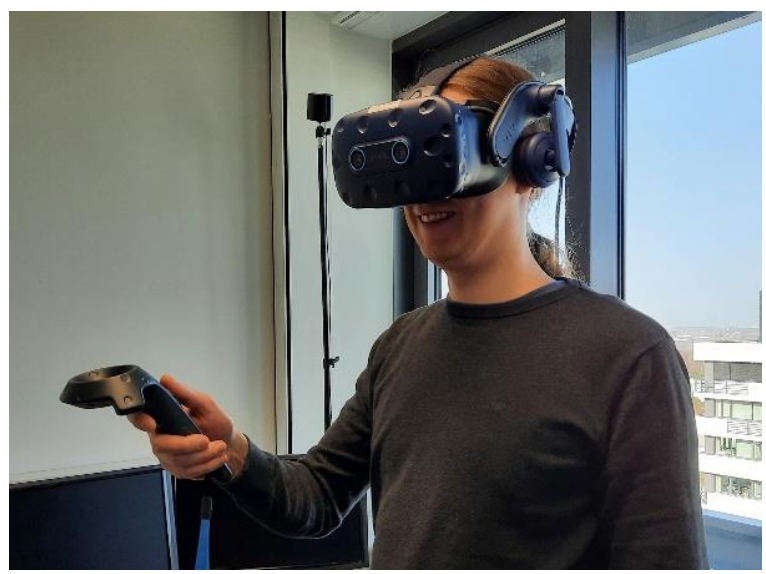

Figure 3. Used VR hardware. Participants were equipped with an HTC Vive Pro HMD and an HTC Vive Controller. 


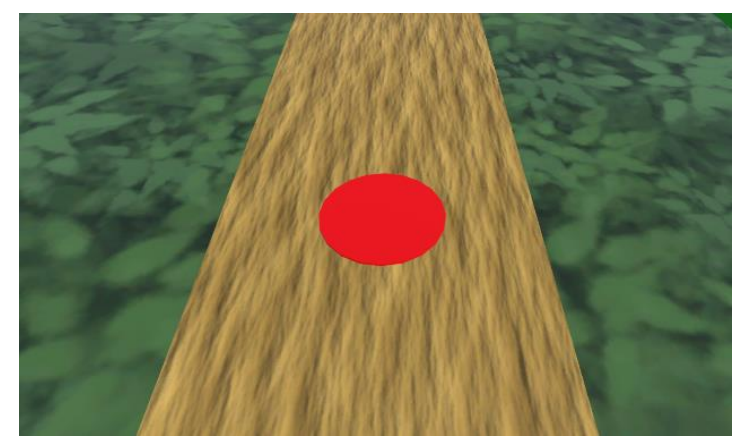

Figure 4. Red target dot. The red dot was used to visualize the target participants had to reach using teleportation or artificial locomotion.

The following experiment scene showed the corridor without the red dot. Participants were asked to use the assigned locomotion technique to travel the same distance as in the previous scene and to press a trigger button on the controller when they thought that they had reached the target (distance invisible target). The same procedure was then repeated for a second time. Hereafter, participants underwent a distance estimation training. The red dot was placed 10 virtual meters away from the starting point of the corridor. Participants were informed about the exact distance of the dot and had to use their assigned type of locomotion to reach it. The training phase was then followed by two additional visible target and invisible target distance estimations. Finally, the whole procedure was repeated with the other locomotion technique (see Table 1).

Table 1. Overview of the experimental procedure. Each participant carried out two visible target and invisible target distance estimations before and after the training using the assigned locomotion technique (either teleportation or artificial locomotion). After completion, the procedure was repeated using the second locomotion technique.

\begin{tabular}{|c|c|c|c|}
\hline \multirow{2}{*}{ Chronological Order } & \multirow{2}{*}{ Training Condition } & \multicolumn{2}{|c|}{ Locomotion Technique } \\
\hline & & Group A & Group B \\
\hline Distance Visible Target & \multirow{4}{*}{ Before Training } & \multirow{9}{*}{ Teleport } & \multirow{9}{*}{$\begin{array}{c}\text { Artificial } \\
\text { Locomotion }\end{array}$} \\
\hline Distance Invisible Target & & & \\
\hline Distance Visible Target & & & \\
\hline Distance Invisible Target & & & \\
\hline Training & & & \\
\hline Distance Visible Target & \multirow{4}{*}{ After Training } & & \\
\hline Distance Invisible Target & & & \\
\hline Distance Visible Target & & & \\
\hline Distance Invisible Target & & & \\
\hline Distance Visible Target & \multirow{4}{*}{ Before Training } & & \multirow{9}{*}{ Teleport } \\
\hline Distance Invisible Target & & & \\
\hline Distance Visible Target & & & \\
\hline Distance Invisible Target & & $\begin{array}{l}\text { Artificial } \\
\text { Locomotion }\end{array}$ & \\
\hline Training & & & \\
\hline Distance Visible Target & \multirow{4}{*}{ After Training } & & \\
\hline Distance Invisible Target & & & \\
\hline Distance Visible Target & & & \\
\hline Distance Invisible Target & & & \\
\hline
\end{tabular}




\subsection{Measures}

Difficulties to estimate distances were assessed based on differences between estimated distances and the correct distance values in (virtual) meters. These differences were calculated as absolute values, as well as directional (real) values. Absolute values were meant to investigate the intensity of distance estimation errors between the different experimental conditions. Directional values were used to indicate whether people underestimated (negative values) or overestimated (positive values) distances, thus whether they stopped before reaching the target or after overshooting the correct distance.

\subsection{Statistics}

After calculating the absolute and directional errors for all distance estimations, error value pairs were aggregated per participant, training condition, and VR locomotion technique. This reduced the amount of error values per participant to four distance estimation error values (two absolute and two directional distance estimation error values) before the training, and four error values after the training.

As indicated in Figure 5, not all response variables were evenly distributed, and multiple outliers were found. Especially for the absolute error values, right-skewedness and outliers were to be expected, because values are by definition limited to the positive range. Visual examination of the plots was supported by analysis of the response variables separately for each combination of experimental conditions (teleportation/artificial locomotion, before training/after training, visible target/invisible target, absolute errors/directional errors) using the Shapiro-Wilk test. Results showed that the response values in 11 of 16 condition combinations were not normally distributed $(p<0.05)$.

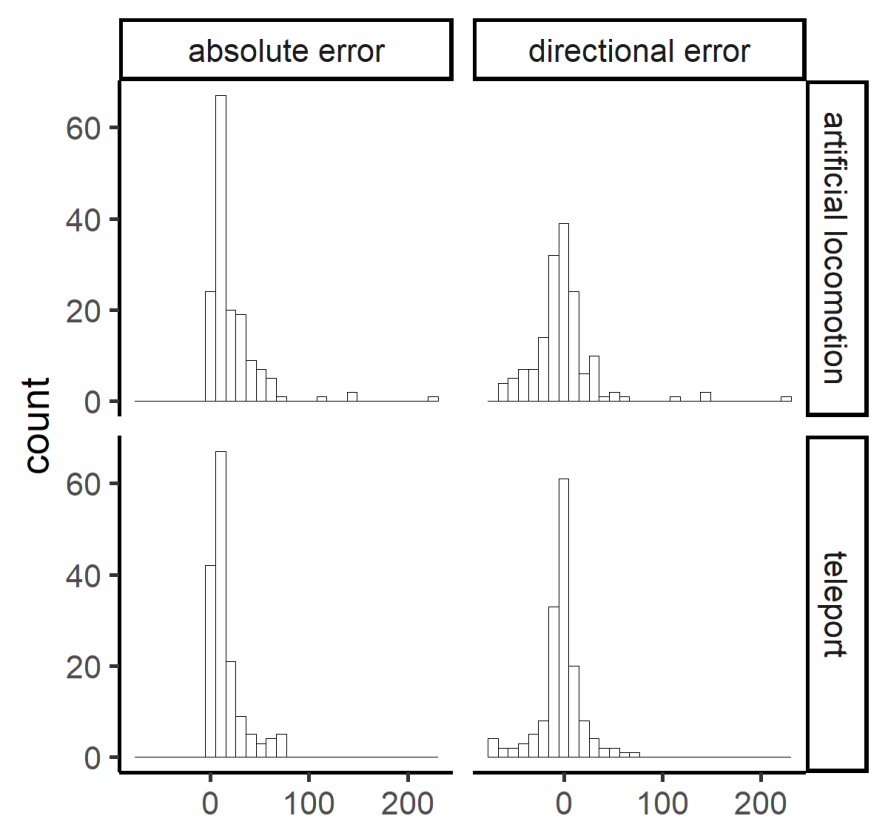

Figure 5. Distribution of the response variables with errors in $\mathrm{m}$. The histograms demonstrate that the response variables were not evenly distributed in all experimental conditions and multiple outliers were found.

Due to the uneven distributions and resulting outliers, we chose nonparametric tests to investigate the potential differences of distance estimation errors implied in our hypotheses. Main and interaction effects of VR locomotion technique and the training condition were investigated separately for the two distance estimation tasks (distance visible target/distance invisible target) and the two error types (absolute errors/directional errors) using Scheirer-Ray-Hare tests. Additionally, as Scheirer-Ray-Hare tests do not account for repeated measures effects, Wilcoxon signed-rank tests were used to verify 
potentially significant main effects concerning the training conditions. Finally, to control whether experience with the teleportation locomotion also improved distance estimations using artificial locomotion, and vice versa, distance estimation errors were compared between the two experiment cycles using Mann-Whitney U tests.

\section{Results}

\subsection{Effects of Locomotion Technique and Training}

The investigation of distance estimations with visible target positions (see Figure 6) showed two significant main effects for absolute distance estimation errors. Absolute errors were on average lower when teleportation locomotion was used $\left(\mathrm{M}_{\text {Teleport }}=22.31 \mathrm{~m}\right.$, $\left.\mathrm{SD}_{\text {Teleport }}=20.58 \mathrm{~m}, \mathrm{M}_{\text {Artificial }}=34.69 \mathrm{~m}, \mathrm{SD}_{\text {Artificial }}=34.43 \mathrm{~m}, \mathrm{H}(1)=9.85, p<0.01\right)$ and after participants received a training $\left(\mathrm{M}_{\text {Before }}=37.62 \mathrm{~m}, \mathrm{SD}_{\text {Before }}=26.82 \mathrm{~m}, \mathrm{M}_{\text {After }}=19.38 \mathrm{~m}\right.$, $\left.\mathrm{SD}_{\text {After }}=28.27 \mathrm{~m}, \mathrm{H}(1)=34.34, p<0.001\right)$. Applying the Wilcoxon signed-rank test to compare absolute distance estimation errors before and after the training also led to significant results $(Z=-5.08, p<0.001)$.
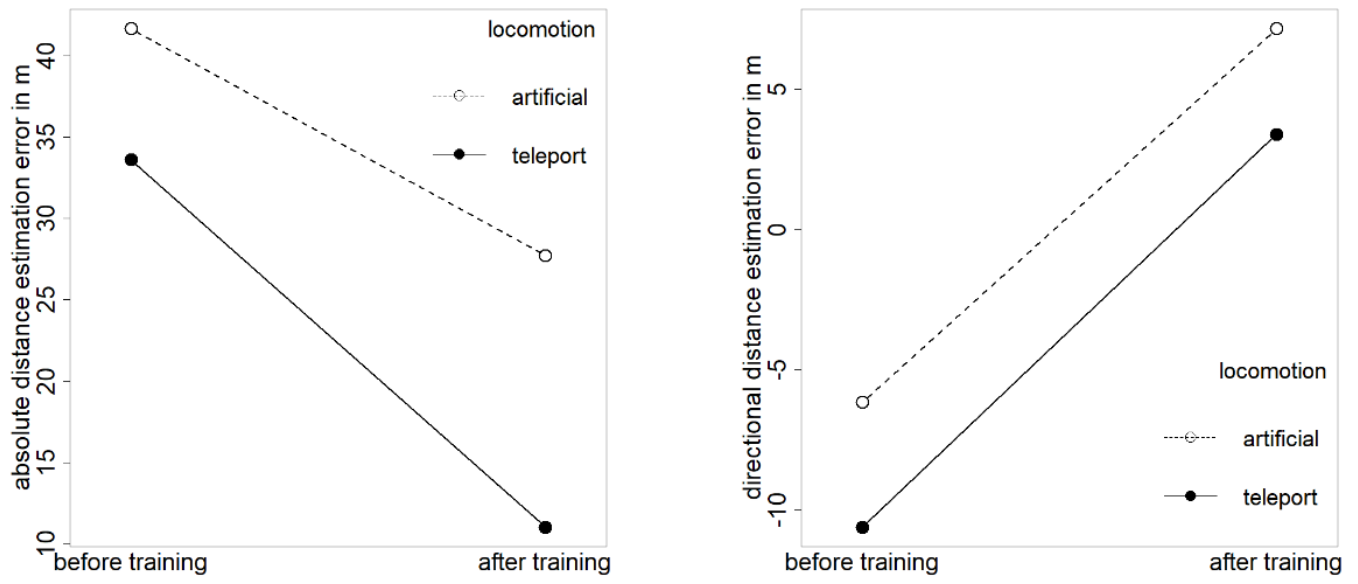

Figure 6. Distance estimation errors for visible targets. The left side of the figure shows the absolute distance estimation errors; the right side shows the directional errors. Absolute distance estimation errors were significantly lower when teleportation locomotion was used $(p<0.01)$. Additionally, absolute distance estimation errors were lower $(p<0.001)$ and estimated distance values were higher $(p<0.01)$ after the training.

A matching significant main effect was found for directional distance estimation errors with visible target positions. Estimated distances were significantly higher after the training $\left(\mathrm{M}_{\text {Before }}=-8.39 \mathrm{~m}, \mathrm{SD}_{\text {Before }}=44.29 \mathrm{~m}, \mathrm{M}_{\text {After }}=5.27 \mathrm{~m}, \mathrm{SD}_{\text {After }}=33.7 \mathrm{~m}, \mathrm{H}(1)=7.53\right.$, $p<0.01)$. Again, using the paired Wilcoxon signed-rank test replicated the significance of mean differences between the training conditions $(Z=3.1, p<0.01)$. However, no significant differences of directional distance estimations with visible target were found between the two locomotion techniques $\left(\mathrm{M}_{\text {Teleport }}=-3.61 \mathrm{~m}, \mathrm{SD}_{\text {Teleport }}=29.78 \mathrm{~m}, \mathrm{M}_{\text {Artificial }}=0.49 \mathrm{~m}\right.$, $\left.\mathrm{SD}_{\text {Artificial }}=47.93 \mathrm{~m}, \mathrm{H}(1)=0.18, p>0.05\right)$.

Concerning both absolute and directional errors of distance estimations with visible target, no significant interaction effects were found between the used locomotion techniques and the training conditions.

Similar to the distance estimations with visible target, absolute errors of distance estimations with invisible target were significantly lower when teleportation locomotion was used (see Figure 7, $\mathrm{M}_{\text {Teleport }}=7.73 \mathrm{~m}, \mathrm{SD}_{\text {Teleport }}=6.93 \mathrm{~m}, \mathrm{M}_{\text {Artificial }}=9.38 \mathrm{~m}$, $\left.\mathrm{SD}_{\text {Artificial }}=5.97 \mathrm{~m}, \mathrm{H}(1)=7.68, p<0.01\right)$ and after participants received a training $\left(\mathrm{M}_{\text {Before }}=10.38 \mathrm{~m}, \mathrm{SD}_{\text {Before }}=7.13 \mathrm{~m}, \mathrm{M}_{\text {After }}=6.73 \mathrm{~m}, \mathrm{SD}_{\text {After }}=5.24 \mathrm{~m}, \mathrm{H}(1)=15, p<0.001\right)$. The significance of the main effect training was also replicated when the Wilcoxon signed- 
rank test was applied $(Z=3.59, p<0.001)$. However, no significant interaction effect was found between the used locomotion techniques and the training conditions.
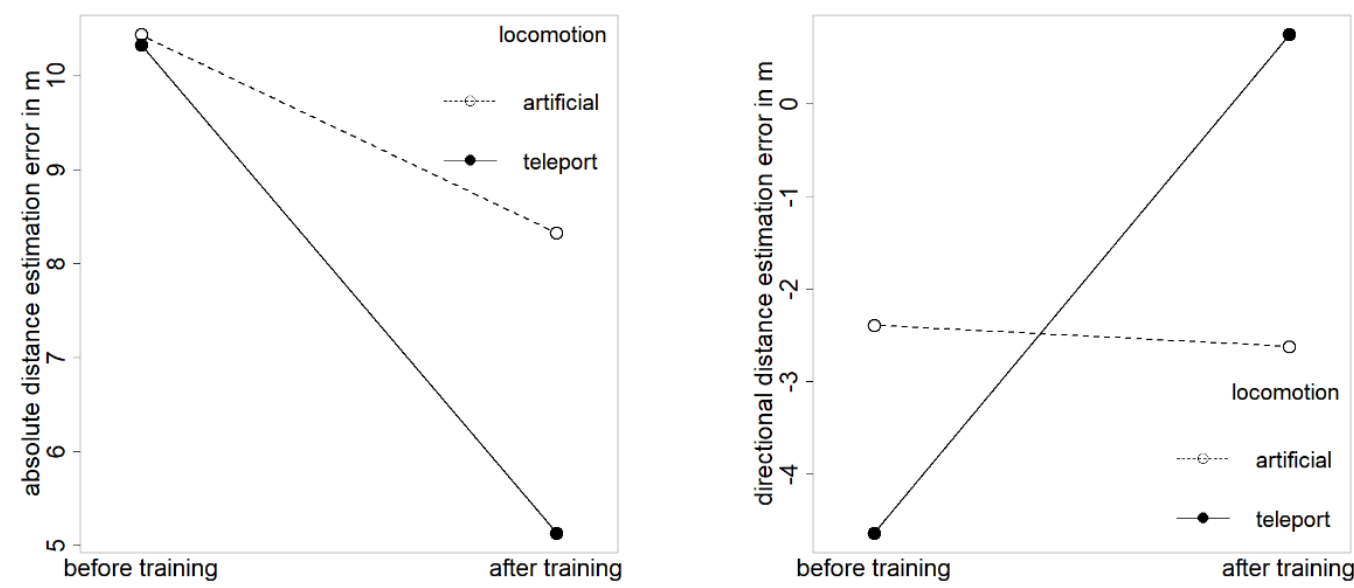

Figure 7. Distance estimation errors for invisible targets. The left side of the figure shows the absolute distance estimation errors; the right side shows the directional errors. After the training, absolute distance estimation errors were significantly lower $(p<0.001)$ and average distance estimations increased $(p<0.05)$. The interaction effect between the training condition and the used locomotion technique on directional distance estimation errors was narrowly not significant $(p=0.052)$. Nevertheless, both graphs indicate that the training affected distance estimations more strongly when the teleportation locomotion was used.

Directional distance estimations with invisible target were significantly higher and errors shifted closer to zero after the training $\left(\mathrm{M}_{\text {Before }}=-3.52 \mathrm{~m}, \mathrm{SD}_{\text {Before }}=10.19 \mathrm{~m}\right.$, $\left.\mathrm{M}_{\text {After }}=-0.94 \mathrm{~m}, \mathrm{SD}_{\text {After }}=7.71 \mathrm{~m}, \mathrm{H}(1)=5.49, p<0.05\right)$. Again, using the Wilcoxon signed-rank test replicated the significant findings of the Scheirer-Ray-Hare test concerning the mean directional distance estimation differences between the training conditions $(Z=2.06 p<0.05)$. Additionally, the interaction effect between the used locomotion techniques and the training conditions was only narrowly above the significance level of 0.05 $(\mathrm{H}(1)=3.77, p=0.052)$. As indicated by the right graph in Figure 7 , the significant main effect of the training conditions seems to be exclusively based on changes in the teleport condition $\left(\mathrm{M}_{\text {Teleport Before }}=-4.64 \mathrm{~m}, \mathrm{SD}_{\text {Teleport Before }}=11.06 \mathrm{~m}, \mathrm{M}_{\text {Teleport After }}=0.75 \mathrm{~m}\right.$, $\mathrm{SD}_{\text {Teleport After }}=5.46 \mathrm{~m}, \quad \mathrm{M}_{\text {Artificial Before }}=-2.4 \mathrm{~m}, \quad \mathrm{SD}_{\text {Artificial Before }}=9.25 \mathrm{~m}$, $\mathrm{M}_{\text {Artificial After }}=-2.62 \mathrm{~m}, \mathrm{SD}_{\text {Artificial After }}=9.2 \mathrm{~m}$ ).

\subsection{Skill Transfer Between Locomotion Techniques}

Comparisons of distance estimations between the two experiment cycles demonstrated a significant training effect across the two locomotion techniques (not to mix up with the before/after training conditions). In the visible target task, absolute distance estimation errors were significantly lower $\left(\mathrm{M}_{\text {First Cycle }}=34.65 \mathrm{~m}, \mathrm{SD}_{\text {First }}\right.$ Cycle $=34.22 \mathrm{~m}$, $\left.\mathrm{M}_{\text {secondCycle }}=22.35 \mathrm{~m}, \mathrm{SD}_{\text {secondCycle }}=20.97 \mathrm{~m}, \mathrm{U}=2340.5, p<0.05\right)$ and mean distance estimation values were significantly higher $\left(\mathrm{M}_{\text {First Cycle }}=-8.02 \mathrm{~m}, \mathrm{SD}_{\text {First Cycle }}=46.97 \mathrm{~m}\right.$, $\left.\mathrm{M}_{\text {secondCycle }}=4.9 \mathrm{~m}, \mathrm{SD}_{\text {secondCycle }}=30.03 \mathrm{~m}, \mathrm{U}=2213, p<0.01\right)$ in the second experiment cycle (see Figure 8). However, no significant differences of distance estimations between the two experiment cycles were found for the invisible target task. 

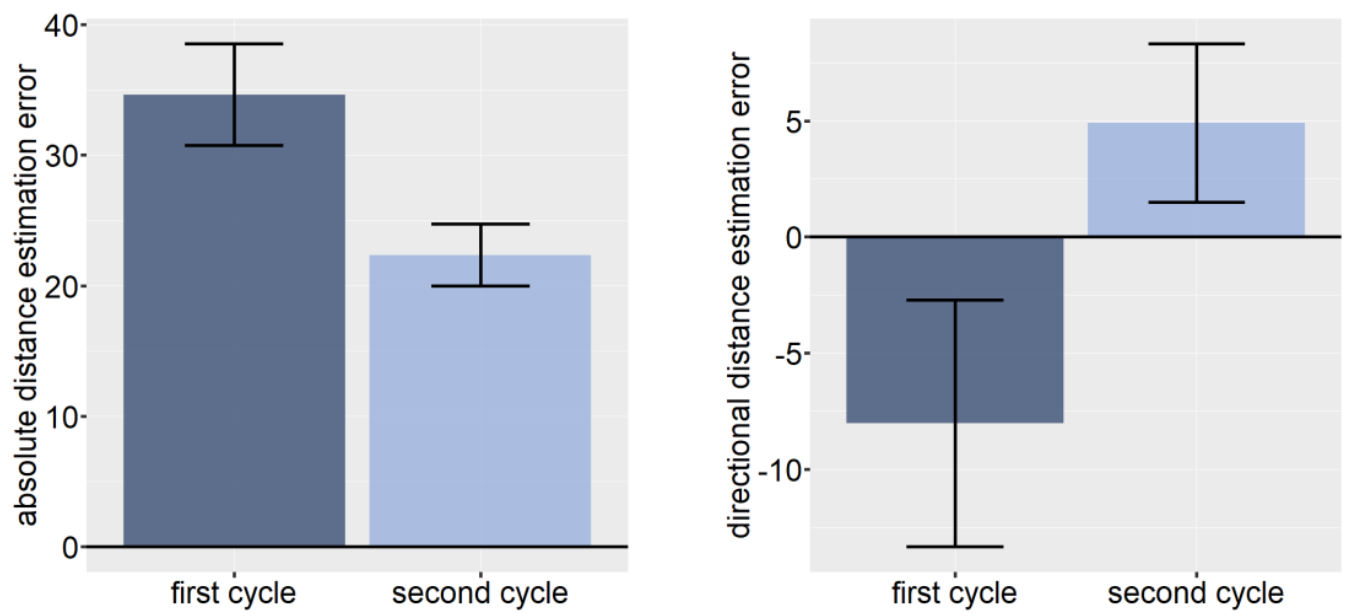

Figure 8. Distance estimation errors between experiment cycles in the visible target task. Mean distance estimation errors were lower in the second experiment cycle. In the first cycle, distances were on average underestimated. In the second cycle, distances were slightly overestimated.

\section{Discussion}

The described experimental results support previous findings and provide further insights into how VR locomotion techniques affect spatial perception in virtual environments. As supposed by our first hypothesis, distances traveled with both artificial locomotion and teleportation locomotion were initially (before the distance estimation training) underestimated (see right chart of Figures 6 and 7). This fits to previous studies that found underestimations of distances travelled with locomotion techniques lacking proprioceptive and vestibular feedback [38,39], as well as studies demonstrating underestimations of distances to objects in extrapersonal space in VR [56-58]. Underestimations and absolute errors of metric distance estimations were more pronounced than underestimations and absolute errors of replicated traveled distances. As already shown in previous studies $[56,63,64]$, making metric distance estimations seems to be challenging, and people tend towards underestimations.

Concerning our second hypothesis, we found no statistical evidence that distance estimations are more inaccurate when teleportation locomotion is used. Underestimations of initial teleportation-based distance estimations were only insignificantly more pronounced than artificial locomotion-based underestimations. Opposed to our expectations, absolute distance estimation errors (underestimations and overestimations combined) were actually significantly higher when artificial locomotion was used (see left chart of Figure 6). These findings may indicate that optic flow self-motion cues, although found to enable distance and time to target estimates $[53,54]$, might be less relevant or helpful for the assessment of traveled distances than other self-motion cues. This may be due to systematic distortions of spatial perception. Multiple studies reported underestimations of optic flow-based distance estimations, especially for longer distances $[38,39,67]$. Furthermore, optic flow speed was found to be perceived as slower than it is [68]. Thus, optic flow has been shown to be an error-prone information source for distance estimations. Based on these studies and our findings, we argue that, despite the availability of an additional self-motion cue (optic flow), using artificial locomotion does not lead to a better distance perception compared to teleportation locomotion. However, it is still unclear whether this is only due to systematic distortions of optic-flow based distance estimations or other differences between artificial and teleportation locomotion. Additional potentially relevant differences such as counting strategies and task duration are discussed below. 
Similarities and differences between artificial locomotion and teleportation locomotion become more distinct when effects of a distance estimation training are compared. In our third hypothesis, we assumed that such a training would improve distance estimations. Indeed, we found that distance estimations with both locomotion types improved after the training (see Figures 6 and 7). This is in line with studies showing that traveling and giving metric estimates of visually perceived or traveled egocentric (self-to-target) distances can be improved by training $[65,66]$. Furthermore, our findings extend these studies by showing similar effects for metric estimations and replications of distances traveled without proprioceptive and vestibular self-motion cues. However, further studies are required to investigate how persistent these improvements are. If performance deteriorates quickly, frequent trainings will be required.

Distinctive differences of training effects on distance estimations were found between metric distance estimations (visible target) and replications of traveled distances (invisible target). Improvements of metric distance estimations were fairly similar for artificial locomotion and teleportation locomotion (see Figure 6). Absolute error values were significantly reduced and directional estimation errors shifted from underestimations to slight overestimations. Thus, participants seem to have overcompensated after the training. Concerning training effects on replications of traveled distances, great differences were found between the two locomotion techniques (see Figure 7). Absolute errors for both locomotion techniques were reduced after the training, but the reduction was more pronounced for teleportation locomotion. Whereas the pronounced underestimations of teleportation-based distance estimations shifted toward minimal overestimations after the training, artificial locomotion-based underestimations showed no difference after the training. We conclude that participants benefited more strongly from a training when they used teleportation to memorize and replicate traveled distances.

It has been addressed above that, besides the availability of optic flow, additional differences between artificial and teleportation locomotion may affect how traveled distances are perceived. Frenz and Lappe [67] found that the duration of a simulated motion affected distance estimations with short motions being underestimated and long motions being overestimated. As participants in our study were not forced to pause between two consecutive teleportations, they were able to travel long distances quickly and faster compared to artificial locomotion. This might explain why underestimations of traveled distances were more pronounced when teleportation locomotion was used. Furthermore, we argue that different counting strategies could have been applied that affected the accuracy of distance estimations. As the artificial locomotion speed was constant, participants could have tried to count the seconds required to reach the $10 \mathrm{~m}$ target in the training condition, subsequently counted the seconds to reach the target in the experimental condition, and then roughly calculated the traveled metric distance [69]. Thus, it appears logical that metric distance estimations improved after participants were provided with a $10 \mathrm{~m}$ reference distance. However, replicating a previously traveled distance did not require the estimation of the metric distance. It was sufficient to count the time required to reach the target. Replicating this distance then only required traveling forward for the same time. This might explain why directional errors of artificial locomotion-based distance estimations did not change after the training. Teleportation locomotion on the other hand is argued to be based on a counting of consecutive teleportations. If consecutive teleportations with similar travel distances are applied, each teleportation can act as a milestone used to subdivide and structure the distance to the target similar to visual milestones provided in a real-world egocentric distance estimation study of Allen and Rashotte [66]. If information about a self-to-(teleportation-)target distance is available, the traveled distance can be calculated based on the number of consecutive teleportations. Therefore, it is understandable that teleportation-based metric distance estimations were more accurate after participants were provided with a $10 \mathrm{~m}$ distance reference during the training. Teleportation-based distance estimations may have furthermore benefited from the fact that the distance of the teleportation target is selected based on the angle of the controller. As 
this angle is determined by the hand and arm posture, motor learning may help to replicate specific teleportation target distances [70,71]. Although metric distance estimations are not necessarily required to replicate previously traveled distances, reducing variations of the hand and arm posture with the distance estimation training may have also helped to assess how many teleportations with similar teleportation target distances are required to reach a specific target location. This might explain why teleportation-based replications of previously traveled distances were significantly more accurate after the training. The different counting strategies might also explain why absolute distance estimation errors were generally higher when artificial locomotion was used despite the availability of optic flow as an additional self-motion cue. Counting seconds may induce higher cognitive demands than counting teleportations, because a constant rhythm must be kept. Increased cognitive demands can then lead to impaired task performance [72,73]. Furthermore, short inattentiveness does not have to cause a miscount of teleportations, but necessarily will affect the accuracy of counted seconds.

Comparing task performance between the two experiment cycles (applied locomotion type) shows that distance estimation skills seem to be transferrable between different locomotion types, as distance estimations were significantly better in the second experiment cycle (see Figure 8). This contradicts findings of Richardson and Waller [65], who found that distance estimation trainings only improved the trained type of distance estimation (egocentric or exocentric). We argue that, in contrast to the study of Richardson and Waller [65], people perceived distances in an unnatural (virtual) environment. As VR consumer technology is still not widely spread, experience of participants with spatial perception in VR is understandably limited. Therefore, it is unclear to what extent improvements in the second experiment cycle can be ascribed to a skill transfer between the two locomotion techniques or to a general habituation to spatial perception in VR space.

Taken together, our findings support our assumption that distance estimations in virtual environments are systematically distorted and are affected by the applied locomotion technique. When an accurate perception of distances in virtual environments is important, it is recommended to use teleportation locomotion instead of artificial locomotion and to provide a short distance estimation training. To what extent the accuracy of distance estimations and the availability of self-motion cues as optic flow affects the formation of mental representations of space in the form of route and location memory requires further investigation.

\section{Summary}

VR locomotion techniques such as artificial locomotion and teleportation have been found to affect how virtual environments are perceived. Both metric distances and replications of previously traveled distances are initially underestimated. People are better at replicating distances than at estimating metric distance values. However, metric estimations can be considerably improved with a distance estimation training. Improvements of replicated distances have also been found, but are less pronounced. After the training, underestimations of metric distances shifted towards slight overestimations, indicating an overcompensation. Contrary to our expectations, the availability of an additional selfmotion cue when artificial locomotion was used (optic flow) did not lead to more accurate distance estimations before or after the training. Concerning both types of investigated distance estimations, improvements after the training were greater when teleportation locomotion was used. We argue that teleportation locomotion allows the application of counting strategies that induce fewer cognitive demands than counting strategies applied during artificial locomotion, because the latter requires keeping track of the travel duration. Furthermore, people seem to be able to transfer distance estimation skills between different VR locomotion techniques. Further studies are required to determine to what extent improvements are caused by the distance estimation training, generally growing experience with visual spatial perception in virtual environments, skill transfer between different VR locomotion techniques, or a combination of these parameters. 
Author Contributions: Conceptualization: Julian Keil, Denise O'Meara, and Frank Dickmann; methodology: Julian Keil; software: Julian Keil and Denise O'Meara; formal analysis: Julian Keil and Annika Korte; investigation: Denise O'Meara; resources: Denise O'Meara and Julian Keil; writing-original draft preparation: Julian Keil and Dennis Edler; writing—review and editing: Julian Keil, Dennis Edler, Frank Dickmann, Annika Korte, and Denise O'Meara; visualization: Julian Keil; supervision: Frank Dickmann; project administration: Frank Dickmann. All authors have read and agreed to the published version of the manuscript.

Funding: This research received no external funding.

Institutional Review Board Statement: The study was conducted according to the guidelines of the Declaration of Helsinki and approved by the Ethics Committee of the Geography Department of the Ruhr University Bochum (19 August 2019).

Informed Consent Statement: Informed consent was obtained from all subjects involved in the study.

Data Availability Statement: The data presented in this study are available on request from the corresponding author.

Conflicts of Interest: The authors declare no conflict of interest.

\section{References}

1. Clarke, K.C.; Johnson, J.M.; Trainor, T. Contemporary American cartographic research: A review and prospective. Cartogr. Geogr. Inf. Sci. 2019, 46, 196-209. [CrossRef]

2. Çöltekin, A.; Griffin, A.L.; Slingsby, A.; Robinson, A.C.; Christophe, S.; Rautenbach, V.; Chen, M.; Pettit, C.; Klippel, A. Geospatial Information Visualization and Extended Reality Displays. In Manual of Digital Earth; Guo, H., Goodchild, M.F., Annoni, A., Eds.; Springer: Singapore, 2020; pp. 229-277. ISBN 978-981-32-9914-6.

3. Montello, D.R.; Fabrikant, S.I.; Davies, C. Cognitive perspectives on cartography and other geographic information visualizations. In Handbook of Behavioral and Cognitive Geography; Montello, D., Ed.; Edward Elgar Publishing: Cheltenham, UK, 2018; pp. 177-196. ISBN 9781784717544.

4. Montello, D.R. Cognitive Map-Design Research in the Twentieth Century: Theoretical and Empirical Approaches. Cartogr. Geogr. Inf. Sci. 2002, 29, 283-304. [CrossRef]

5. Uttal, D.H.; Wellman, H.M. Young children's representation of spatial information acquired from maps. Dev. Psychol. 1989, 25, 128-138. [CrossRef]

6. Münzer, S.; Zimmer, H.D.; Schwalm, M.; Baus, J.; Aslan, I. Computer-assisted navigation and the acquisition of route and survey knowledge. J. Environ. Psychol. 2006, 26, 300-308. [CrossRef]

7. Dickmann, F.; Edler, D.; Bestgen, A.-K.; Kuchinke, L. Exploiting Illusory Grid Lines for Object-Location Memory Performance in Urban Topographic Maps. Cartogr. J. 2017, 54, 242-253. [CrossRef]

8. De Cock, L.; Ooms, K.; van de Weghe, N.; Vanhaeren, N.; de Maeyer, P. User Preferences on Route Instruction Types for Mobile Indoor Route Guidance. ISPRS Int. J. Geo-Inf. 2019, 8, 482. [CrossRef]

9. Lokka, I.E.; Çöltekin, A.; Wiener, J.; Fabrikant, S.I.; Röcke, C. Virtual environments as memory training devices in navigational tasks for older adults. Sci. Rep. 2018, 8, 10809. [CrossRef] [PubMed]

10. Slater, M.; Linakis, V.; Usoh, M.; Kooper, R. Immersion, Presence, and Performance in Virtual Environments: An Experiment with Tri-Dimensional Chess. In Proceedings of the ACM Symposium on Virtual Reality Software and Technology, VRST'96, Hong Kong, 1-4 July 1996; Green, M., Fairchild, K., Zyda, M., Eds.; ACM Press: New York, NY, USA, 1996; pp. 163-172, ISBN 0897918258.

11. Slater, M. Immersion and the illusion of presence in virtual reality. Br. J. Psychol. 2018, 109, 431-433. [CrossRef]

12. Hruby, F.; Ressl, R.; de La Borbolla del Valle, G. Geovisualization with immersive virtual environments in theory and practice. Int. J. Digit. Earth 2019, 12, 123-136. [CrossRef]

13. Edler, D.; Husar, A.; Keil, J.; Vetter, M.; Dickmann, F. Virtual Reality (VR) and Open Source Software: A Workflow for Constructing an Interactive Cartographic VR Environment to Explore Urban Landscapes. KN J. Cartogr. Geogr. Inf. 2018, 68, 5-13. [CrossRef]

14. Edler, D.; Keil, J.; Wiedenlübbert, T.; Sossna, M.; Kühne, O.; Dickmann, F. Immersive VR Experience of Redeveloped Postindustrial Sites: The Example of "Zeche Holland" in Bochum-Wattenscheid. KN J. Cartogr. Geogr. Inf. 2019, 69, 267-284. [CrossRef]

15. Keil, J.; Edler, D.; Schmitt, T.; Dickmann, F. Creating Immersive Virtual Environments Based on Open Geospatial Data and Game Engines. J. Cartogr. Geogr. Inf. 2021, 1-13. [CrossRef]

16. Çöltekin, A.; Oprean, D.; Wallgrün, J.O.; Klippel, A. Where are we now? Re-visiting the Digital Earth through human-centered virtual and augmented reality geovisualization environments: Re-visiting the Digital Earth through human-centered virtual and augmented reality geovisualization environments. Int. J. Digit. Earth 2019, 12, 119-122. [CrossRef] 
17. Hruby, F. The Sound of Being There: Audiovisual Cartography with Immersive Virtual Environments. J. Cartogr. Geogr. Inf. 2019, 69, 19-28. [CrossRef]

18. Schmohl, S.; Tutzauer, P.; Haala, N. Stuttgart City Walk: A Case Study on Visualizing Textured DSM Meshes for the General Public Using Virtual Reality. PFG-J. Photogramm. Remote Sens. Geoinf. Sci. 2020, 88, 147-154. [CrossRef]

19. Zhao, J.; Wallgrün, J.O.; La Femina, P.C.; Normandeau, J.; Klippel, A. Harnessing the power of immersive virtual realityVisualization and analysis of 3D earth science data sets. Geo-Spat. Inf. Sci. 2019, 22, 237-250. [CrossRef]

20. Jamei, E.; Mortimer, M.; Seyedmahmoudian, M.; Horan, B.; Stojcevski, A. Investigating the Role of Virtual Reality in Planning for Sustainable Smart Cities. Sustainability 2017, 9, 2006. [CrossRef]

21. Ma, Y.; Wright, J.; Gopal, S.; Phillips, N. Seeing the invisible: From imagined to virtual urban landscapes. Cities 2020, $98,102559$. [CrossRef]

22. Lütjens, M.; Kersten, T.; Dorschel, B.; Tschirschwitz, F. Virtual Reality in Cartography: Immersive 3D Visualization of the Arctic Clyde Inlet (Canada) Using Digital Elevation Models and Bathymetric Data. Multimodal Technol. Interact. 2019, 3, 9. [CrossRef]

23. Edler, D.; Kühne, O.; Jenal, C.; Vetter, M.; Dickmann, F. Potenziale der Raumvisualisierung in Virtual Reality (VR) für die sozialkonstruktivistische Landschaftsforschung. J. Cartogr. Geogr. Inf. 2018, 68, 245-254. [CrossRef]

24. Berger, M.; Bill, R. Combining VR Visualization and Sonification for Immersive Exploration of Urban Noise Standards. Multimodal Technol. Interact. 2019, 3, 34. [CrossRef]

25. Kersten, T.P.; Deggim, S.; Tschirschwitz, F.; Lindstaedt, M.; Hinrichsen, N. Segeberg 1600—Eine Stadtrekonstruktion in Virtual Reality. KN J. Cartogr. Geogr. Inf. 2018, 68, 183-191. [CrossRef]

26. Büyüksalih, G.; Kan, T.; Özkan, G.E.; Meriç, M.; Isın, L.; Kersten, T.P. Preserving the Knowledge of the Past Through Virtual Visits: From 3D Laser Scanning to Virtual Reality Visualisation at the Istanbul Çatalca İnceğiz Caves. PFG-J. Photogramm. Remote Sens. Geoinf. Sci. 2020, 88, 133-146. [CrossRef]

27. Carbonell-Carrera, C.; Saorín, J.L. Geospatial Google Street View with Virtual Reality: A Motivational Approach for Spatial Training Education. ISPRS Int. J. Geo-Inf. 2017, 6, 261. [CrossRef]

28. Šašinka, Č.; Stachoň, Z.; Sedlák, M.; Chmelík, J.; Herman, L.; Kubíček, P.; Šašinková, A.; Doležal, M.; Tejkl, H.; Urbánek, T.; et al. Collaborative Immersive Virtual Environments for Education in Geography. ISPRS Int. J. Geo-Inf. 2019, 8, 3. [CrossRef]

29. Hruby, F.; Castellanos, I.; Ressl, R. Cartographic Scale in Immersive Virtual Environments. J. Cartogr. Geogr. Inf. 2020, 21, 399. [CrossRef]

30. Liang, Z.; Zhou, K.; Gao, K. Development of Virtual Reality Serious Game for Underground Rock-Related Hazards Safety Training. IEEE Access 2019, 7, 118639-118649. [CrossRef]

31. Boletsis, C.; Cedergren, J.E. VR Locomotion in the New Era of Virtual Reality: An Empirical Comparison of Prevalent Techniques. Adv. Hum.-Comput. Interact. 2019, 2019, 1-15. [CrossRef]

32. Ruddle, R.A.; Volkova, E.; Bülthoff, H.H. Walking improves your cognitive map in environments that are large-scale and large in extent. ACM Trans. Comput.-Hum. Interact. 2011, 18, 1-20. [CrossRef]

33. Coomer, N.; Bullard, S.; Clinton, W.; Williams-Sanders, B. Evaluating the effects of four VR locomotion methods: Joystick, arm-cycling, point-tugging, and teleporting. In Proceedings of the SAP'18: 15th ACM Symposium on Applied Perception, Vancouver, BC, Canada, 10-11 August 2018; Grimm, C., Willemsen, P., Eds.; Association for Computing Machinery: New York, NY, USA, 2018; pp. 1-8.

34. Cherep, L.A.; Lim, A.F.; Kelly, J.W.; Acharya, D.; Velasco, A.; Bustamante, E.; Ostrander, A.G.; Gilbert, S.B. Spatial cognitive implications of teleporting through virtual environments. J. Exp. Psychol. Appl. 2020, 26, 480-492. [CrossRef]

35. Kelly, J.W.; Ostrander, A.G.; Lim, A.F.; Cherep, L.A.; Gilbert, S.B. Teleporting through virtual environments: Effects of path scale and environment scale on spatial updating. IEEE Trans. Vis. Comput. Graph. 2020, 26, 1841-1850. [CrossRef] [PubMed]

36. Paris, R.; Klag, J.; Rajan, P.; Buck, L.; McNamara, T.P.; Bodenheimer, B. How Video Game Locomotion Methods Affect Navigation in Virtual Environments. In Proceedings of the ACM Symposium on Applied Perception 2019, SAP'19: ACM Symposium on Applied Perception 2019, Barcelona, Spain, 19-20 September 2019; Neyret, S., Kokkinara, E., Franco, M.G., Hoyet, L., Cunningham, D.W., Świdrak, J., Eds.; ACM: New York, NY, USA, 2019; pp. 1-7, ISBN 9781450368902.

37. Epstein, R.A.; Patai, E.Z.; Julian, J.B.; Spiers, H.J. The cognitive map in humans: Spatial navigation and beyond. Nat. Neurosci. 2017, 20, 1504-1513. [CrossRef] [PubMed]

38. Campos, J.L.; Butler, J.S.; Bülthoff, H.H. Contributions of visual and proprioceptive information to travelled distance estimation during changing sensory congruencies. Exp. Brain Res. 2014, 232, 3277-3289. [CrossRef]

39. Campos, J.L.; Butler, J.S.; Bülthoff, H.H. Multisensory integration in the estimation of walked distances. Exp. Brain Res. 2012, 218, 551-565. [CrossRef]

40. Cheng, Z.; Gu, Y. Vestibular System and Self-Motion. Front. Cell. Neurosci. 2018, 12, 456. [CrossRef]

41. Lappe, M.; Bremmer, F.; van den Berg, A.V. Perception of self-motion from visual flow. Trends Cogn. Sci. 1999, 3, 329-336. [CrossRef]

42. Koenderink, J.J. Optic flow. Vis. Res. 1986, 26, 161-179. [CrossRef]

43. Montello, D.R. Navigation. In The Cambridge Handbook of Visuospatial Thinking; Shah, P., Miyake, A., Eds.; Cambridge University Press: Cambridge, UK, 2012; pp. 257-294. ISBN 9780511610448.

44. Dietz, V. Proprioception and locomotor disorders. Nat. Rev. Neurosci. 2002, 3, 781-790. [CrossRef]

45. Lopez, C.; Blanke, O. The thalamocortical vestibular system in animals and humans. Brain Res. Rev. 2011, 67, 119-146. [CrossRef] 
46. Angelaki, D.E.; Cullen, K.E. Vestibular system: The many facets of a multimodal sense. Annu. Rev. Neurosci. 2008, 31, 125-150. [CrossRef] [PubMed]

47. Langbehn, E.; Lubos, P.; Bruder, G.; Steinicke, F. Bending the Curve: Sensitivity to Bending of Curved Paths and Application in Room-Scale VR. IEEE Trans. Vis. Comput. Graph. 2017, 23, 1389-1398. [CrossRef]

48. Shewaga, R.; Uribe-Quevedo, A.; Kapralos, B.; Alam, F. A Comparison of Seated and Room-Scale Virtual Reality in a Serious Game for Epidural Preparation. IEEE Trans. Emerg. Top. Comput. 2020, 8, 218-232. [CrossRef]

49. Langbehn, E.; Lubos, P.; Steinicke, F. Evaluation of Locomotion Techniques for Room-Scale VR: Joystick, Teleportation, and Redirected Walking. In Proceedings of the Virtual Reality International Conference-Laval Virtual on-VRIC'18, the Virtual Reality International Conference-Laval Virtual, Laval, France, 4-6 April 2018; Richir, S., Ed.; ACM Press: New York, NY, USA, 2018; pp. 1-9, ISBN 9781450353816.

50. Schnack, A.; Wright, M.J.; Holdershaw, J.L. Does the locomotion technique matter in an immersive virtual store environmentComparing motion-tracked walking and instant teleportation. J. Retail. Consum. Serv. 2021, 58, 1-10. [CrossRef]

51. Porter, J.; Robb, A. An Analysis of Longitudinal Trends in Consumer Thoughts on Presence and Simulator Sickness in VR Games. In Proceedings of the Annual Symposium on Computer-Human Interaction in Play. CHI PLAY '19: The Annual Symposium on Computer-Human Interaction in Play, Barcelona, Spain, 22-25 October 2019; Arnedo, J., Nacke, L.E., Vanden Abeele, V., Toups, Z.O., Eds.; ACM: New York, NY, USA, 2019; pp. 277-285, ISBN 9781450366885.

52. Bhandari, J.; MacNeilage, P.; Folmer, E. Teleportation without Spatial Disorientation Using Optical Flow Cues. In Proceedings of the 44th Graphics Interface Conference, Toronto, ON, Canada, 8-11 May 2018; Batty, C., Ed.; Canadian Human-Computer Communications Society: Waterloo, ON, Canada, 2018. ISBN 978-0-9947868-3-8.

53. McLeod, R.W.; Ross, H.E. Optic-flow and cognitive factors in time-to-collision estimates. Perception 1983, 12, 417-423. [CrossRef] [PubMed]

54. Redlick, F.P.; Jenkin, M.; Harris, L.R. Humans can use optic flow to estimate distance of travel. Vis. Res. 2001, 41, 213-219. [CrossRef]

55. Previc, F.H. The Neuropsychology of 3-D Space. Psychol. Bull. 1998, 124, 123-164. [CrossRef]

56. Armbrüster, C.; Wolter, M.; Kuhlen, T.; Spijkers, W.; Fimm, B. Depth Perception in Virtual Reality: Distance Estimations in Periand Extrapersonal Space. Cyberpsychol. Behav. 2008, 11, 9-15. [CrossRef] [PubMed]

57. Naceri, A.; Chellali, R.; Hoinville, T. Depth Perception within Peripersonal Space Using Head-Mounted Display. Presence 2011, 20, 254-272. [CrossRef]

58. Ng, A.K.T.; Chan, L.K.Y.; Lau, H.Y.K. Depth Perception in Virtual Environment: The Effects of Immersive System and Freedom of Movement. In Proceedings of the VAMR 2016: Virtual, Augmented and Mixed Reality. International Conference on Virtual, Augmented and Mixed Reality, Toronto, ON, Canada, 17-22 July 2016; Lackey, S., Shumaker, R., Eds.; Springer: Cham, Switzerland, 2016. [CrossRef]

59. Witmer, B.G.; Sadowski, W.J. Nonvisually Guided Locomotion to a Previously Viewed Target in Real and Virtual Environments. Hum. Factors 1998, 40, 478-488. [CrossRef]

60. Murgia, A.; Sharkey, P.M. Estimation of Distances in Virtual Environments Using Size Constancy. Int. J. Virtual Real. 2019, 8, 67-74. [CrossRef]

61. Willemsen, P.; Gooch, A.A. Perceived egocentric distances in real, image-based, and traditional virtual environments. In Proceedings of the IEEE Virtual Reality 2002, Orlando, FL, USA, 24-28 March 2002; IEEE Computer Society: Washington, DC, USA, 2002; pp. 275-276, ISBN 0-7695-1492-8.

62. Messing, R.; Durgin, F.H. Distance Perception and the Visual Horizon in Head-Mounted Displays. ACM Trans. Appl. Percept. 2005, 2, 234-250. [CrossRef]

63. Keil, J.; Korte, A.; Ratmer, A.; Edler, D.; Dickmann, F. Augmented Reality (AR) and Spatial Cognition: Effects of Holographic Grids on Distance Estimation and Location Memory in a 3D Indoor Scenario. PFG-J. Photogramm. Remote Sens. Geoinf. Sci. 2020, 88, 165-172. [CrossRef]

64. Stefanucci, J.K.; Proffitt, D.R.; Banton, T.; Epstein, W. Distances appear different on hills. Percept. Psychophys. 2005, 67, 1052-1060. [CrossRef]

65. Richardson, A.R.; Waller, D. The effect of feedback training on distance estimation in virtual environments. Appl. Cognit. Psychol. 2005, 19, 1089-1108. [CrossRef]

66. Allen, G.L.; Rashotte, M.A. Training metric accuracy in distance estimation skill: Pictures versus words. Appl. Cognit. Psychol. 2006, 20, 171-186. [CrossRef]

67. Frenz, H.; Lappe, M. Absolute travel distance from optic flow. Vis. Res. 2005, 45, 1679-1692. [CrossRef] [PubMed]

68. Banton, T.; Stefanucci, J.; Durgin, F.; Fass, A.; Proffitt, D. The Perception of Walking Speed in a Virtual Environment. Presence: Teleoper. Virtual Environ. 2005, 14, 394-406. [CrossRef]

69. Frenz, H.; Lappe, M.; Kolesnik, M.; Bührmann, T. Estimation of travel distance from visual motion in virtual environments. ACM Trans. Appl. Percept. 2007, 4,1-18. [CrossRef]

70. Magill, R.A.; Anderson, D. Motor Learning and Control. Concepts and Applications, 10th ed.; McGraw-Hill: New York, NY, USA, 2014; ISBN 978-0-07-802267-8. 
71. Brooks, V.B. Motor control. How posture and movements are governed. Phys. Ther. 1983, 63, 664-673. [CrossRef]

72. Paas, F.; Tuovinen, J.E.; Tabbers, H.; van Gerven, P.W.M. Cognitive Load Measurement as a Means to Advance Cognitive Load Theory. Educ. Psychol. 2003, 38, 63-71. [CrossRef]

73. Kirschner, P.A. Cognitive load theory: Implications of cognitive load theory on the design of learning. Learn. Instr. 2002, 12, 1-10. [CrossRef] 